Journal of Mathematics and Statistics 8 (1): 157-164, 2012

ISSN 1549-3644

(C) 2012 Science Publications

\title{
Policy Decisions for a Price Dependent Demand Rate Inventory Model with Progressive Payments Scheme
}

\author{
${ }^{1}$ Mukesh Kumar and ${ }^{2}$ Rajat Kumar \\ Department of Mathematics, \\ ${ }^{1}$ Graphic Era University, Dehradun (Uttarakhand), India \\ ${ }^{2}$ Krishna Institute of Management and Technology, Moradabad, India
}

\begin{abstract}
Problem statement: In this proposed research, we developed an inventory model to formulate an optimal ordering policies for supplier who offers progressive permissible delay periods to the retailer to settle his/her account. We assumed that the annual demand rate as a decreasing function of price with constant rate of deterioration and time-varying holding cost. Shortages in inventory are allowed which is completely backlogged. Approach: The main objective of this study to frame an inventory model in real life situations. In this study, we introduced a new idea of trade credits, namely, the supplier charges the retailer progressive interest rates if the retailer prolongs its unpaid balance. By offering progressive interest rates to the retailers, a supplier, can secure competitive market advantage over the competitors and possibly improve market share profit. This study has two main purposes, first the mathematical model of an inventory system are establish under the above conditions and second demonstrate that the optimal solution not only exists but also feasible. We developed theoretical results to obtain the optimal replenishment interval by examine the explicit condition. An algorithm is given to find the flow of optimal ordering policy. Results: The results is illustrated with the help of numerical example using Mathematica software and the optimal solution of the problem is $\mathrm{Z}\left(\mathrm{p}, \mathrm{T}_{1}\right)=$ 76.8586 at $\left(\mathrm{p}, \mathrm{T}_{1}\right)=(0.952656,0.128844)$. Conclusion: We proposed an algorithm to find the optimal ordering policy. A numerical study has been performed to observe the sensitivity of the effect of demand parameter changes.
\end{abstract}

Key words: Linear holding cost, progressive permissible delay, deterioration rate and shortages

\section{INTRODUCTION}

In the traditional Economic Order Quantity (EOQ) model, it is assumed that the retailer pays for the goods as soon as it is received by the system. However, in practice, the supplier offers a retailer a delay of fixed time period for setting the amount owed to him. Usually, there is no interest charge if the outstanding amount is paid within the credit period. However, if the payment is not paid in full by the end of the credit period, then interest is charged on the outstanding amount. Goyal (1985)eveloped an EOQ model under conditions of permissible delay in payments extended Goyal (1985) model by allowing shortages. Mandal and Phaujdar (1988) developed an inventory model by including interest earned from the sales revenue on the stoke remaining beyond the settlement period. Aggarwal and Jaggi (1995) extended Goyal's model for deteriorating items because the loss due to deterioration cannot be ignored. Jamal et al. (1997) generalized the model to allow for shortage and deterioration. Liao et al. (2000); Chang and Dye (2001); Teng (2002); Teng et al. (2005) and Hwang and Shinn (1997) developed the model with permissible delay in period. Chang et al. (2010) Developed an Optimal replenishment policies for non-instantaneous deteriorating items with stockdependent demand.

In the progressive trade credit period, retailer settles the outstanding amount by first credit period. Hence, the supplier does not charge any interest. Supplier charges an interest at rate $\mathrm{Ic}_{1}$ on the un-paid balance if retailer pays after first credit period but before second period offered by supplier to retailer. If retailer settles his amount after second credit period, then supplier charges to retailer an interest at rate $\mathrm{Ic}_{2}$ on un-paid balance $\left(\mathrm{Ic}_{1}<\mathrm{Ic}_{2}\right)$. By assuming progressive trade credits to the retailer supplier can secure competitive market advantage and improve market share. Goyal et al. (2007) developed an inventory model with constant demand rate and deterioration rate under progressive payment scheme. Soni and Shah (2008) developed a model for stoke-dependent

Corresponding Author: Mukesh Kumar, Department of Mathematics Graphic Era University, Dehradun (Uttarakhand), India 
demand rate under progressive payment scheme. Singh et al. (2008) extended Soni and Shah (2008) model by allowing shortages and variable holding cost. This fact attracted a number of researchers to drive inventory modals on price dependent demand rate patterns. Presented an inventory model for items havinf the demand rate is constant and variable deterioration rate under the trade credits. Some of the related works in this area are by Haley and Higgins (1973); Wee (1995); Chung and Tsai (2001); Teng (2002) and Teng et al. (2005).

In this study, we address the issues relating to progressive credit period relating to the retailer to settle his account. We developed a mathematical model when the demand rate, as a decreasing function of price and shortage which are fully backlogged with time varying holding cost. We assume that the supplier offers two progressive credit periods to the retailer to settle the account. The net profit is maximized by optimization technique. An algorithm is presented to derive the retailer's optimal solution.

Fundamental assumptions and notations: The following assumptions are used to develop the model:

- The inventory system deals with the single item.

- Replenishment rate is finite

- $\quad$ Shortage are allowed and completely back logged.

- Lead time is zero

- The annual demand, as a decreasing function of price; we get $D(p)=\alpha p^{-\beta}$, Where $\alpha>0$ and $\beta>1$. p denotes selling price of the item during the cycle time and a decision variable

- If the retailer pays by $\mathrm{M}$, then suppliers does not charge to the retailer. If the retailer pays after $\mathbf{M}$ and before $\mathrm{N}(\mathrm{N}>\mathrm{M})$, he can keep the difference in the unit sale price and unit cost in an interest bearing account at the rate of Ie /unit/year. During $[\mathrm{M}, \mathrm{N}]$, the supplier charges the retailer an interest rate $\mathrm{Ic}_{1}$ /unit/year. If the retailer pays after $\mathrm{N}$, then supplier charges the retailer an interest rate of $\mathrm{Ic}_{2}$ /unit/year $\left(\mathrm{Ic}_{2}>\mathrm{Ic}_{1}\right)$ on unpaid balance

The notations are as follows:
$\mathrm{Ic}_{1}=$ Interests charge per $\not \subset$ in stock per year by supplier when retailer pays during $[\mathrm{M}, \mathrm{N}]$

$\mathrm{Ic}_{1} \quad=$ Interests charge per $\not \delta$ in stock per year by supplier when retailer pays during $[\mathrm{N}, \mathrm{T}]$ $\left(\mathrm{Ic}_{2}>\mathrm{Ic}_{1}\right)$

$\mathrm{I}_{\mathrm{e}} \quad=$ Interest earned $/ \not /$ year

$\mathrm{T}=$ Replenishment cycle

$\theta=$ Deterioration rate, $0<\theta<<1$.

$\mathrm{HC}=$ Holding cost $\mathrm{h}(\mathrm{t})$ per timr unit and it is time dependent and is assumed as:

$\mathrm{h}(\mathrm{t}) \quad=\alpha+\beta \mathrm{t}$, where $\alpha>0, \beta>0$.

OC $=$ Ordering cost $/$ time unit

IE $\quad=$ Interest earned/time unit

IC = Interest charged/time unit

$\mathrm{Q}(\mathrm{t}) \quad=$ On hand inventory at time $\left(0 \leq \mathrm{t} \leq \mathrm{T}_{1}\right)$

GR = Gross revenue

SC $=$ Shortage cost/time unit

$\mathrm{Z}\left(\mathrm{p}, \mathrm{T}_{1}\right)=$ Total net profit/time unit

Formulation of mathematical model Eq 1 and 2:

$\frac{\mathrm{d}}{\mathrm{dt}} \mathrm{Q}(\mathrm{t})+\theta \mathrm{Q}(\mathrm{t})=-\mathrm{D}, \quad 0 \leq \mathrm{t} \leq \mathrm{T}_{1}$

$\frac{\mathrm{d}}{\mathrm{dt}} \mathrm{Q}(\mathrm{t})=-\mathrm{D}, \quad \mathrm{T}_{1} \leq \mathrm{t} \leq \mathrm{T}$

With boundary conditions, $\mathrm{Q}(0)=\mathrm{Q}, \mathrm{Q}\left(\mathrm{T}_{1}\right)=0$, consequently, the solution of the above Eq. 3-5 are:

$\mathrm{Q}(\mathrm{t})=\frac{\mathrm{D}}{\mathrm{Q}}\left[\mathrm{e}^{\left(\mathrm{T}_{1}-\mathrm{t}\right)}-1\right], \quad 0 \leq \mathrm{t} \leq \mathrm{T}_{1}$

$\mathrm{Q}(\mathrm{t})=\mathrm{D}\left(\mathrm{T}_{1}-\mathrm{t}\right), \quad \mathrm{T}_{1} \leq \mathrm{t} \leq \mathrm{T}$

And the order quantity is $\mathrm{Q}=\frac{\mathrm{D}}{\theta}\left(\mathrm{e}^{\theta \mathrm{T}_{1}}-1\right)$

The cost components per unit time are as follows Eq. 6:

Ordering Cost $(\mathrm{OC})=\frac{\mathrm{A}}{\mathrm{T}}$

Inventory holding cost Eq. 7:

$$
\begin{aligned}
& \mathrm{HC}=\frac{\mathrm{h}}{\mathrm{T}} \int_{0}^{\mathrm{T}_{1}} \mathrm{Q}(\mathrm{t}) \mathrm{dt}=\frac{\mathrm{h}}{\mathrm{T}} \int_{0}^{\mathrm{T}_{1}} \frac{\mathrm{D}}{\theta}\left[\mathrm{e}^{\theta\left(\mathrm{T}_{1}-\mathrm{t}\right)}-1\right] \mathrm{dt} \\
& =\frac{\mathrm{hD}}{\theta^{2} \mathrm{~T}}\left[\mathrm{e}^{\theta \mathrm{T}_{1}}-\theta \mathrm{T}_{1}-1\right]
\end{aligned}
$$


The deterioration cost in the time interval $\left[0, \mathrm{~T}_{1}\right]$ is Eq. 8:

$$
\mathrm{DC}=\frac{\mathrm{CD}}{\theta \mathrm{T}}\left[\mathrm{e}^{\theta \mathrm{T}_{1}}-\theta \mathrm{T}_{1}-1\right]
$$

Shortage cost occurs during the period $\left[\mathrm{T}_{1}, \mathrm{~T}\right]$ is given by Eq. 9 and 10:

$$
\begin{aligned}
& S C=\frac{S}{T} \int_{T_{1}}^{T}-Q(t) d t=\frac{S}{T} \int_{T_{1}}^{T}-D\left(T_{1}-t\right) d t \\
& =\frac{S D}{T}\left[\frac{T^{2}+T_{1}^{2}}{2}-T_{1} T\right]
\end{aligned}
$$

Gross revenue $\mathrm{GR}=(\mathrm{pc}) \mathrm{D}(\mathrm{p})$

Regarding interest charged and interest earned based on the length of the cycle time $\mathrm{T}_{1}$, three cases arise:

Case 1: $\mathrm{T}_{1} \leq \mathrm{M}$, Case 2: $\mathrm{M}<\mathrm{T}_{1}<\mathrm{N}$ and Case 3: $\mathrm{T}_{1} \geq \mathrm{N}$. Case 1: $\mathrm{T}_{1} \leq \mathrm{M}$;

Here, Retailer sells $\mathrm{Q}$ units during $\left(0, \mathrm{~T}_{1}\right)$ and paying for $\mathrm{CQ}$ units in full to the supplier at time $\mathrm{M}$ $\geq \mathrm{T}_{1}$, so interest charges are zero, i.e. Eq. 11:

$\mathrm{IC}_{1}=0$

Retailer deposits the revenue in an interest bearing account at the rate of $\mathrm{Ie} / \not /$ /year. Therefore interest earned $\mathrm{IE}_{1}$, per year is Eq. 12:

$$
\begin{aligned}
& \mathrm{IE}_{1}=\frac{P \mathrm{I}_{\mathrm{e}}}{\mathrm{T}}\left[\int_{0}^{\mathrm{T}_{1}} \mathrm{Q}(\mathrm{t}) \cdot \mathrm{tdt}+\left(\mathrm{M}-\mathrm{T}_{1}\right) \int_{0}^{\mathrm{T}_{1}} \mathrm{Q}(\mathrm{t}) \mathrm{dt}\right] \\
& =\frac{\mathrm{PI}}{\mathrm{T}}\left[\frac{\mathrm{D}}{\theta^{3}}\left\{\mathrm{e}^{\theta \mathrm{T}_{1}}-\theta \mathrm{T}_{1}-\frac{\theta^{2} \mathrm{~T}_{1}^{2}}{2}-1\right\} \begin{array}{c}
\frac{\mathrm{D}\left(\mathrm{M}-\mathrm{T}_{1}\right)}{\theta^{2}} \\
\left\{\mathrm{e}^{\theta \mathrm{T}_{1}}-\theta \mathrm{T}_{1}-1\right\}
\end{array}\right]
\end{aligned}
$$

To maximize the net profit at $\mathrm{T}_{1}=\mathrm{T}_{1}{ }^{*}$ and $\mathrm{p}=\mathrm{p}^{*}$, here $\mathrm{T}$ is fixed for one year planning horizon provided. The net profit Eq. 13:

$$
\begin{aligned}
& \mathrm{Z}_{1}\left(p, T_{1}\right)=G R-O C-H C-D C-I_{1}+I_{1}-S C \\
& =(p-c) D(p)-\frac{A}{T}+\frac{h D(p)}{\theta^{2} T}\left[e^{\theta T_{1}}-\theta T_{1}-1\right] \\
& -\frac{c D(p)}{\theta T}\left[e^{\theta T_{1}}-\theta T_{1}-1\right]-\frac{S D(p)}{T}\left(\frac{T^{2}+T_{1}^{2}}{2}-T_{1}\right) \\
& +\frac{P I_{e}}{T}\left[\frac{D(p)}{\theta^{3}}\left\{e^{\theta T_{1}}-\theta T_{1}-\frac{\theta^{2} T_{1}^{2}}{2}-1\right\}\right. \\
& \left.+\frac{D(p)\left(M-T_{1}\right)}{\theta^{2}}\left\{e^{\theta T_{1}}-\theta T_{1}-1\right\}\right]
\end{aligned}
$$

$\mathrm{p}$ and $\mathrm{T}_{1}$ are continuous variables. Hence the optimal values of $\mathrm{p}$ and $\mathrm{T}_{1}$ can be obtained by setting Eq. 14, 15 :

$$
\begin{aligned}
& \frac{\partial}{\partial \mathrm{p}} \mathrm{Z}_{1}\left(\mathrm{p}, \mathrm{T}_{1}\right)=\alpha \mathrm{p}^{-\beta}+\alpha \beta c \mathrm{p}^{-\beta-1}-\alpha \beta \mathrm{p}^{-\beta}+\frac{\alpha \beta \mathrm{p}^{-\beta-1} \mathrm{~h}}{\theta^{2} \mathrm{~T}} \\
& \left\{\mathrm{e}^{\theta \mathrm{T}_{1}}-\theta \mathrm{T}_{1}-1\right\}+\frac{\mathrm{c} \alpha \beta \mathrm{p}^{-\beta-1}}{\theta \mathrm{T}}\left\{\mathrm{e}^{\theta \mathrm{T}_{1}}-\theta \mathrm{T}_{1}-1\right\}+\frac{\mathrm{S} \alpha \beta \mathrm{p}^{-\beta-1}}{\mathrm{~T}} \\
& \left(\frac{\mathrm{T}^{2}+\mathrm{T}_{1}^{2}}{2}-\mathrm{TT}_{1}\right)+\frac{\mathrm{pIe}}{\mathrm{T}}\left[\frac{-\alpha \beta \mathrm{p}^{-\beta-1}}{\theta^{3}}\left\{\begin{array}{c}
\mathrm{e}^{\theta \mathrm{T}_{1}}-\theta \mathrm{T}_{1} \\
-\frac{\theta^{2} \mathrm{~T}_{1}^{2}}{2}-1
\end{array}\right\}\right. \\
& \left.-\frac{\alpha \beta \mathrm{p}^{-\beta-1}\left(\mathrm{M}-\mathrm{T}_{1}\right)}{\theta^{2}}\left\{\mathrm{e}^{\theta \mathrm{T}_{1}}-\theta \mathrm{T}_{1}-1\right\}\right]+\frac{\mathrm{Ie}}{\mathrm{T}} \\
& {\left[\frac{\mathrm{D}(\mathrm{p})}{\theta^{3}}\left\{\mathrm{e}^{\theta \mathrm{T}_{1}}-\theta \mathrm{T}_{1}-\frac{\theta^{2} \mathrm{~T}_{1}^{2}}{2}-1\right\}\right.} \\
& \left.+\frac{\mathrm{D}(\mathrm{p})\left(\mathrm{M}-\mathrm{T}_{1}\right)}{\theta^{2}}\left\{\mathrm{e}^{\theta \mathrm{T}_{1}}-\theta \mathrm{T}_{1}-1\right\}\right]=0
\end{aligned}
$$

And:

$$
\begin{aligned}
& \frac{\partial}{\partial \mathrm{T}_{1}} \mathrm{Z}_{1}\left(\mathrm{p}, \mathrm{T}_{1}\right)=0 \\
& \Rightarrow-\frac{\mathrm{hD}}{\theta \mathrm{T}}\left\{\mathrm{e}^{\theta \mathrm{T}_{1}}-1\right\}-\frac{\mathrm{cD}}{\mathrm{T}}\left\{\mathrm{e}^{\theta \mathrm{T}_{1}}-1\right\}-\frac{\mathrm{SD}}{\mathrm{T}}\left\{\mathrm{T}_{1}-\mathrm{T}\right\}+\frac{\mathrm{pI}}{\mathrm{T}} \\
& {\left[\frac{\mathrm{D}}{\theta^{2}}\left\{\mathrm{e}^{\theta \mathrm{T}_{1}}-\theta \mathrm{T}_{1}-1\right\}+\frac{\mathrm{D}\left(\mathrm{M}-\mathrm{T}_{1}\right)}{\mathrm{Q}}\left\{\mathrm{e}^{\theta \mathrm{T}_{1}}-1\right\}-\frac{\mathrm{D}}{\theta^{2}}\right]=0} \\
& \left.\Rightarrow\left\{\frac{\mathrm{pIe}}{\mathrm{T}}-1\right\} \frac{\mathrm{D}\left\{\mathrm{e}^{\theta \mathrm{T}_{1}}-\theta \mathrm{e}_{1}-1\right\}}{\mathrm{T}_{1}}-\theta \mathrm{T}_{1}-1\right\} \\
& \theta^{2}+\frac{\mathrm{pIeD}\left(\mathrm{M}-\mathrm{T}_{1}\right)}{\theta \mathrm{T}}\left\{\mathrm{e}^{\theta \mathrm{T}_{1}}-1\right\} \\
& -\left(\frac{\mathrm{h}}{\theta}+\mathrm{c}\right) \frac{\mathrm{D}\left\{\mathrm{e}^{\theta \mathrm{T}_{1}}-1\right\}}{\mathrm{T}}-\frac{\mathrm{SD}\left(\mathrm{T}_{1}-\mathrm{T}\right)}{\mathrm{T}}=0
\end{aligned}
$$

To maximize the net profit, provided Eq. 16:

$$
\mathrm{rt}-\mathrm{S} 2<0
$$

Where:

$$
\begin{aligned}
& r=\frac{\partial^{2}}{\partial p^{2}} \mathrm{Z}_{1}\left(\mathrm{p}, \mathrm{T}_{1}\right)=-\alpha \beta \mathrm{p}^{-\beta-1}-\alpha \beta(\beta+1) c \mathrm{p}^{\beta-2}+\alpha \beta^{2} \mathrm{p}^{-\beta-1} \\
& -\frac{\alpha \beta(\beta+1) \mathrm{p}^{-\beta-2} \mathrm{~h}}{\theta^{2} \mathrm{~T}}\left\{\mathrm{e}^{\theta \mathrm{T}_{1}}-\theta \mathrm{T}_{1}-1\right\}-\frac{\operatorname{co} \beta(\beta+1) \mathrm{p}^{-\beta-2}}{\theta \mathrm{T}} \\
& \left\{\mathrm{e}^{\theta \mathrm{T}_{1}}-\theta \mathrm{T}_{1}-1\right\}-\frac{\mathrm{S} \alpha \beta(\beta+1) \mathrm{e}^{-\beta-2}}{\mathrm{~T}}\left\{\mathrm{~T}^{2}+\mathrm{T}_{1}^{2}-\mathrm{TT}_{1}\right\} \\
& +\frac{\mathrm{pIe}}{\mathrm{T}}\left[\begin{array}{c}
\left.\frac{\alpha \beta(\beta+1) \mathrm{e}^{-\beta-2}}{\theta^{3}}\left\{\mathrm{e}^{\theta \mathrm{T}_{1}}-\theta \mathrm{T}_{1}-\frac{\theta^{2} \mathrm{~T}_{1}^{2}}{2}-1\right\}\right] \\
\left.+\frac{\alpha \beta(\beta+1)\left(\mathrm{M}-\mathrm{T}_{1}\right) \mathrm{p}^{-\beta-2}}{\theta^{2}}\left\{\mathrm{e}^{\theta \mathrm{T}_{1}}-\theta \mathrm{T}_{1}-1\right\}\right]
\end{array}\right.
\end{aligned}
$$




$$
\begin{aligned}
& -\frac{\alpha \beta p^{-\beta-1} \mathrm{Ie}}{\mathrm{T}}\left[\begin{array}{l}
\frac{1}{\theta^{3}}\left\{\mathrm{e}^{\theta \mathrm{T}_{1}}-\theta \mathrm{T}_{1}-\frac{\theta^{2} \mathrm{~T}_{1}^{2}}{2}-1\right\}+ \\
\frac{\left(\mathrm{M}-\mathrm{T}_{1}\right)}{\theta^{2}}\left\{\mathrm{e}^{\theta \mathrm{T}_{1}}-\theta \mathrm{T}_{1}-1\right\}
\end{array}\right] \\
& \mathrm{t}=\frac{\partial^{2}}{\partial \mathrm{T}_{1}^{2}} \mathrm{Z}_{1}\left(\mathrm{p}, \mathrm{T}_{1}\right)=\left(\frac{\mathrm{pI}}{\mathrm{T}}-1\right) \frac{\mathrm{D}}{\theta}\left\{\mathrm{e}^{\theta \mathrm{T}_{1}}-1\right\}+\frac{\mathrm{pIeD}\left(\mathrm{M}-\mathrm{T}_{1}\right)}{\mathrm{T}} \\
& \mathrm{e}^{\theta \mathrm{T}_{1}}+\frac{\mathrm{pIeD}}{\theta \mathrm{T}}\left\{\mathrm{e}^{\theta \mathrm{T}_{1}}-1\right\}-\left(\frac{\mathrm{h}}{\theta}+\mathrm{c}\right) \frac{\mathrm{D} \theta \mathrm{e}^{\theta \mathrm{T}_{1}}}{\mathrm{~T}}-\frac{\mathrm{SD}}{\mathrm{T}}
\end{aligned}
$$

And:

$$
\begin{aligned}
& \mathrm{s}=\frac{\partial^{2}}{\partial \mathrm{T}_{1} \partial \mathrm{p}} \mathrm{Z}_{1}\left(\mathrm{p}, \mathrm{T}_{1}\right)=-\left(\frac{\mathrm{pI}}{\mathrm{T}}-1\right) \frac{\alpha \beta \mathrm{p}^{-\beta-1}}{\theta^{2}}\left\{\mathrm{e}^{\theta \mathrm{T}_{1}}-\theta \mathrm{T}_{1}-1\right\} \\
& +\frac{\mathrm{DIe}}{\theta^{2} \mathrm{~T}}\left\{\mathrm{e}^{\theta \mathrm{T}_{1}}-\theta \mathrm{T}_{1}-1\right\}+\frac{\mathrm{DIe}\left(\mathrm{M}-\mathrm{T}_{1}\right)}{\theta \mathrm{T}}\left\{\mathrm{e}^{\theta \mathrm{T}_{1}}-1\right\} \\
& -\frac{\alpha \beta \mathrm{p}^{-\beta} \mathrm{Ie}\left(\mathrm{M}-\mathrm{T}_{1}\right)}{\theta \mathrm{T}}\left\{\mathrm{e}^{\theta \mathrm{T}_{1}}-1\right\}+\left(\frac{\mathrm{h}}{\theta}+\mathrm{c}\right) \frac{\alpha \beta \mathrm{p}^{-\beta-1}}{\mathrm{~T}} \\
& \left\{\mathrm{e}^{\theta \mathrm{T}_{1}}-1\right\}+\frac{\mathrm{S} \alpha \beta \mathrm{e}^{-\beta-1}\left(\mathrm{~T}_{1}-\mathrm{T}\right)}{\mathrm{T}}
\end{aligned}
$$

Case 2: $\mathrm{M}<\mathrm{T}_{1}<\mathrm{N}$.

The interest earned, $\mathrm{IE}_{2}$ during $[0, \mathrm{M}]$ is Eq. 17:

$$
\begin{aligned}
& \mathrm{IE}_{2}=\mathrm{PI}_{\mathrm{e}} \int_{0}^{\mathrm{M}} \mathrm{D}(\mathrm{p}) \cdot \mathrm{t} d \mathrm{t}=\mathrm{PI}_{\mathrm{e}} \int_{0}^{\mathrm{M}} \alpha \mathrm{p}^{-\beta} \cdot \mathrm{t} d \mathrm{t} \\
& =\mathrm{PI}_{\mathrm{e}} \alpha \mathrm{p}^{-\beta} \frac{\mathrm{M}^{2}}{2}=\frac{1}{2} \alpha \mathrm{p}^{1-\beta} \mathrm{I}_{\mathrm{e}} \mathrm{M}^{2}
\end{aligned}
$$

The retailer pay for $\mathrm{Q}$ units purchased at time $\mathrm{t}=0$ at the rate of $\mathrm{C} \not \delta$ /unit to the supplier during $[0, \mathrm{M}]$. The retailer sells $\mathrm{D}(\mathrm{p}) \mathrm{M}$ units at selling price P/unit. So, he has generated revenue of p.D (p) $\mathrm{M}+\mathrm{IE}_{2}$

\section{Then two sub cases may arise:}

Sub case: 2.1: Let p.D (p).M+IE $2 \geq C Q$, i.e., retailer has enough money to pay for all $\mathrm{Q}$ units procured. Then interest charge will be Eq. 18:

$$
\mathrm{IC}_{2.1}=0
$$

And interest earned per unit time is Eq. 19:

$$
\mathrm{IE}_{2.1}=\frac{\mathrm{IE}_{2}}{\mathrm{~T}}=\frac{\alpha \mathrm{p}^{1-\beta} \mathrm{IeM}^{2}}{2 \mathrm{~T}}
$$

The net profit Eq. 20:

$$
\begin{aligned}
& \mathrm{Z}_{2.1}\left(\mathrm{p}, \mathrm{T}_{1}\right)=\mathrm{GR}-\mathrm{OC}-\mathrm{HC}-\mathrm{DC}-\mathrm{SC}-\mathrm{Ic}_{2.1}+\mathrm{IE}_{2.1} \\
& \mathrm{Z}_{2.1}\left(\mathrm{p}, \mathrm{T}_{1}\right)=(\mathrm{p}-\mathrm{c}) \alpha \mathrm{p}^{-\beta}-\frac{\mathrm{A}}{\mathrm{T}}-\frac{\mathrm{h} \alpha \mathrm{p}^{-\beta}}{\theta^{2} \mathrm{~T}}\left\{\mathrm{e}^{\theta \mathrm{T}_{1}}-\theta \mathrm{T}_{1}-1\right\} \\
& -\frac{\mathrm{c} \alpha \mathrm{p}^{-\beta}}{\theta \mathrm{T}}\left\{\mathrm{e}^{\theta \mathrm{T}_{1}}-\theta \mathrm{T}_{1}-1\right\}-\frac{\mathrm{S} \alpha \mathrm{p}^{-\beta}}{\mathrm{T}} \\
& \left(\frac{\mathrm{T}^{2}+\mathrm{T}_{1}^{2}}{2}-\mathrm{TT}_{1}\right)+\frac{\alpha \mathrm{p}^{1-\beta} \mathrm{IeM}^{2}}{2 \mathrm{~T}}
\end{aligned}
$$

The optimal values of $\mathrm{p}=\mathrm{p}_{2.1}$ and $\mathrm{T} 1=\mathrm{T}_{2.1}$ are solutions of Eq. 21 and 22:

$$
\begin{aligned}
& \frac{\partial}{\partial \mathrm{p}} \mathrm{Z}_{2.1}\left(\mathrm{p}, \mathrm{T}_{1}\right)=\alpha \mathrm{p}^{-\beta}+\alpha \beta c \mathrm{p}^{-\beta-1}-\alpha \beta \mathrm{p}^{-\beta}+\frac{\alpha \beta \mathrm{p}^{-\beta-1} \mathrm{~h}}{\theta^{2} \mathrm{~T}} \\
& \left\{\mathrm{e}^{\theta \mathrm{T}_{1}}-\theta \mathrm{T}_{1}-1\right\}+\frac{\mathrm{c} \alpha \beta \mathrm{p}^{-\beta-1}}{\theta \mathrm{T}}\left\{\mathrm{e}^{\theta \mathrm{T}_{1}}-\theta \mathrm{T}_{1}-1\right\} \\
& +\frac{\mathrm{S} \alpha \beta \mathrm{p}^{-\beta-1}}{\mathrm{~T}}\left(\frac{\mathrm{T}^{2}+\mathrm{T}_{1}^{2}}{2}-\mathrm{TT}_{1}\right)+\frac{\alpha(1-\beta) \mathrm{p}^{-\beta} \mathrm{IeM}^{2}}{2 \mathrm{~T}}=0
\end{aligned}
$$

And:

$$
\begin{aligned}
& \frac{\partial}{\partial \mathrm{T}_{1}} \mathrm{Z}_{2.1}\left(\mathrm{p}, \mathrm{T}_{1}\right)=\frac{-\mathrm{hD}}{\theta \mathrm{T}}\left\{\mathrm{e}^{\theta \mathrm{T}_{1}}-1\right\}-\frac{\mathrm{CD}}{\mathrm{T}}\left\{\mathrm{e}^{\theta \mathrm{T}_{1}}-1\right\}-\frac{\mathrm{SD}}{\mathrm{T}}\left(\mathrm{T}_{1}-\mathrm{T}\right)=0 \\
& =\frac{-\mathrm{D}}{\mathrm{T}}\left\{\frac{\mathrm{h}}{\theta}+1\right\}\left\{\mathrm{e}^{\theta \mathrm{T}_{1}}-1\right\}-\frac{\mathrm{SD}}{\mathrm{T}}\left(\mathrm{T}_{1}-\mathrm{T}\right)=0
\end{aligned}
$$
23-26:

For maximizing the total net profit, provided Eq.

$$
\mathrm{r}_{2.1} \mathrm{t}_{2.1}-\mathrm{s}_{2.1}^{2}<0
$$

Where:

$$
\begin{aligned}
& \mathrm{r}_{2.1}=\frac{\partial^{2}}{\partial \mathrm{p}^{2}} \mathrm{Z}_{2,1}\left(\mathrm{p}, \mathrm{T}_{1}\right)=-\alpha \beta \mathrm{p}^{-\beta-1}-\alpha \beta(\beta+1) \mathrm{cp}^{-\beta-2} \\
& +\alpha \beta^{2} \mathrm{p}^{-\beta-1}-\frac{\alpha \beta(\beta+1) \mathrm{p}^{-\beta-2} \mathrm{~h}}{\theta^{2} \mathrm{~T}}\left\{\mathrm{e}^{\theta \mathrm{T}_{1}}-\theta \mathrm{T}_{1}-1\right\} \\
& -\frac{\operatorname{co} \beta(\beta+1) \mathrm{p}^{-\beta-2}}{\theta \mathrm{T}}\left\{\mathrm{e}^{\theta \mathrm{T}_{1}}-\theta \mathrm{T}_{1}-1\right\}-\frac{\mathrm{S} \alpha \beta(\beta+1) \mathrm{p}^{-\beta-2}}{\mathrm{~T}} \\
& \left(\frac{\mathrm{T}^{2}+\mathrm{T}_{1}^{2}}{2}-\mathrm{TT}_{1}\right)-\frac{\alpha \beta(1-\beta) \mathrm{p}^{-\beta-1} \mathrm{IeM}^{2}}{2 \mathrm{~T}} \\
& \mathrm{t}_{2.1}=\frac{\partial^{2}}{\partial \mathrm{T}_{1}^{2}} \mathrm{Z}_{2.1}\left(\mathrm{p}, \mathrm{T}_{1}\right)=\frac{-\mathrm{D}}{\mathrm{T}}\left\{\frac{\mathrm{h}}{\theta}+1\right\} \theta \mathrm{e}^{\theta \mathrm{T}_{1}}- \\
& \frac{\mathrm{SD}}{\mathrm{T}}=\frac{-\mathrm{D}}{\mathrm{T}}\left[\mathrm{S}+(\mathrm{h}+\theta) \mathrm{e}^{\theta \mathrm{T}_{1}}\right]
\end{aligned}
$$

And:

$$
\begin{aligned}
& \mathrm{s}_{2.1}=\frac{\partial^{2}}{\partial \mathrm{T}_{1} \partial \mathrm{p}} \mathrm{Z}_{2.1}\left(\mathrm{p}, \mathrm{T}_{1}\right)=\frac{\alpha \beta \mathrm{p}^{-\beta-1} \mathrm{~h}}{\theta \mathrm{T}}\left\{\mathrm{e}^{\theta \mathrm{T}_{1}}-1\right\} \\
& +\frac{\mathrm{c} \alpha \beta \mathrm{p}^{-\beta-1}}{\mathrm{~T}}\left\{\mathrm{e}^{\theta \mathrm{T}_{1}}-1\right\}+\frac{\mathrm{S} \alpha \beta \mathrm{p}^{-\beta-1}}{\mathrm{~T}}\left\{\mathrm{~T}_{1}-\mathrm{T}\right\} \\
& =\frac{\alpha \beta \mathrm{p}^{-\beta-1}}{\mathrm{~T}}\left\{\frac{\mathrm{h}}{\theta}+1\right\}\left\{\mathrm{e}^{\theta \mathrm{T}_{1}}-1\right\}+\frac{\mathrm{S} \alpha \beta \mathrm{p}^{-\beta-1}}{\mathrm{~T}}\left\{\mathrm{~T}_{1}-\mathrm{T}\right\}
\end{aligned}
$$


Sub Case: 2.2: $\quad$ Let p.D (p) $M+\mathrm{IE}_{2}<\mathrm{CQ}$. Here retailer will have to pay interest on unpaid balance.

$\mathrm{U}_{1}=\mathrm{cD}(\mathrm{p})-\left[\mathrm{p} . \mathrm{D}\left(\mathrm{pM}+\mathrm{IE}_{2}\right)\right]$ at the rate of $\mathrm{Ic}_{1}$ at time $\mathrm{M}$ to the supplier. Then interest paid, $\mathrm{Ic}_{2,2}$ per unit time is given by Eq. 27:

$$
\begin{aligned}
& \mathrm{IC}_{2.2}=\frac{\mathrm{U}_{1}^{2} \mathrm{Ic}_{1}}{\operatorname{PD}(\mathrm{p}) \cdot \mathrm{T}} \int_{\mathrm{M}}^{\mathrm{T}_{1}} \mathrm{Q}(\mathrm{t}) \mathrm{dt} \\
& =\frac{\mathrm{U}_{1}^{2} \mathrm{Ic}_{1}}{\mathrm{PT} \theta^{2}}\left[\mathrm{e}^{\theta\left(\mathrm{T}_{1}-\mathrm{M}\right)}+\theta\left(\mathrm{M}-\mathrm{T}_{1}\right)-1\right]
\end{aligned}
$$

And Interest earned Eq. 28:

$$
\mathrm{IE}_{2.2}=\frac{\mathrm{IE}_{2}}{\mathrm{~T}}=\frac{\alpha \mathrm{p}^{1-\beta} \mathrm{I}_{\mathrm{e}} \mathrm{M}^{2}}{2 \mathrm{~T}}
$$

Net profit $Z_{2.2}\left(p, T_{1}\right)$ is given by Eq. 29:

$$
\begin{aligned}
& \mathrm{Z}_{2.2}\left(\mathrm{p}, \mathrm{T}_{1}\right)=\mathrm{GR}-\mathrm{OC}-\mathrm{HC}-\mathrm{DC}-\mathrm{SC}-\mathrm{Ic}_{2.2}+\mathrm{IE}_{2.2} \\
& =(\mathrm{p}-\mathrm{c}) \alpha \mathrm{p}^{-\beta}-\frac{\mathrm{A}}{\mathrm{T}}-\frac{\mathrm{h} \alpha \mathrm{p}^{-\beta}}{\theta^{2} \mathrm{~T}}\left[\mathrm{e}^{\theta \mathrm{T}_{1}}-\theta \mathrm{T}_{1}-1\right]-\frac{c \alpha \mathrm{p}^{-\beta}}{\theta \mathrm{T}} \\
& {\left[\mathrm{e}^{\theta \mathrm{T}_{1}}-\theta \mathrm{T}_{1}-1\right]-\frac{\mathrm{S} \alpha \mathrm{p}^{-\beta}}{\mathrm{T}}\left(\frac{\mathrm{T}^{2}+\mathrm{T}_{1}^{2}}{2}-\mathrm{TT}_{1}\right)} \\
& -\frac{\mathrm{U}_{1}^{2} \mathrm{Ic}_{1}}{\mathrm{pT} \theta^{2}}\left\{\mathrm{e}^{\theta\left(\mathrm{T}_{1}-\mathrm{M}\right)}+\theta\left(\mathrm{M}-\mathrm{T}_{1}\right)-1\right\}+\frac{\alpha \mathrm{p}^{1-\beta} \mathrm{IeM}^{2}}{2 \mathrm{~T}}
\end{aligned}
$$

The optimal values of $\mathrm{p}=\mathrm{p}_{2.2}$ and $\mathrm{T}_{1}=\mathrm{T}_{2.2}$ are solutions of Eq. 30 and 31:

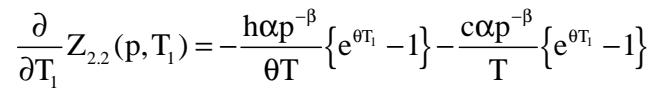

$$
\begin{aligned}
& -\frac{{\mathrm{S} \alpha \mathrm{p}^{-\beta}}_{\mathrm{T}}}{\mathrm{T}}\left\{\mathrm{T}_{1}-\mathrm{T}\right\}-\frac{\mathrm{U}_{1}^{2} \mathrm{Ic}_{1}}{\mathrm{pT} \theta}\left\{\mathrm{e}^{\theta\left(\mathrm{T}_{1}-\mathrm{M}\right)}-1\right\}=0
\end{aligned}
$$

And:

$$
\begin{aligned}
& \frac{\partial}{\partial \mathrm{p}} \mathrm{Z}_{2.2}\left(\mathrm{p}, \mathrm{T}_{1}\right)=\alpha \mathrm{p}^{-\beta}-\alpha \beta(\mathrm{p}-\mathrm{c}) \mathrm{p}^{-\beta-1}+\frac{h \alpha \beta \mathrm{p}^{-\beta-1}}{\theta^{2} \mathrm{~T}} \\
& \left\{\mathrm{e}^{\theta \mathrm{T}_{1}}-\theta \mathrm{T}_{1}-1\right\}+\frac{\mathrm{c} \alpha \beta \mathrm{p}^{-\beta-1}}{\theta \mathrm{T}}\left\{\mathrm{e}^{\theta \mathrm{T}_{1}}-\theta \mathrm{T}_{1}-1\right\} \\
& +\frac{\mathrm{S} \alpha \beta \mathrm{p}^{-\beta-1}}{\mathrm{~T}}\left\{\frac{\mathrm{T}^{2}+\mathrm{T}_{1}^{2}}{2}-\mathrm{TT}_{1}\right\}+\frac{\mathrm{U}_{1}^{2} \mathrm{Ic}_{1}}{\mathrm{p}^{2} \mathrm{~T}^{2}}\left\{\begin{array}{l}
\mathrm{e}^{\theta\left(\mathrm{T}_{1}-\mathrm{M}\right)} \\
+\theta\left(\mathrm{M}-\mathrm{T}_{1}\right)-1
\end{array}\right\} \\
& +\frac{\alpha(1-\beta) \mathrm{p}^{-\beta} \mathrm{IeM}^{2}}{2 \mathrm{~T}}=0
\end{aligned}
$$

At $\mathrm{p}=\mathrm{p}_{2.2}$ and $\mathrm{T} 1=\mathrm{T}_{2.2}$ maximized the net profit provided Eq. 32-35:

$$
\mathrm{r}_{2.2} \mathrm{t}_{2.2}-\mathrm{s}_{2.2}^{2}<0
$$

Where:

$$
\begin{aligned}
& \mathrm{r}_{2.2}=\frac{\partial^{2}}{\partial \mathrm{p}^{2}} \mathrm{Z}_{2.2}\left(\mathrm{p}, \mathrm{T}_{1}\right)=-\alpha \beta \mathrm{p}^{-\beta-1}+\alpha \beta(\beta+1)(\mathrm{p}-\mathrm{c}) \\
& \mathrm{p}^{-\beta-2}-\alpha \beta \mathrm{p}^{-\beta-1}-\frac{\mathrm{h} \alpha \beta(\beta+1) \mathrm{p}^{-\beta-2}}{\theta^{2} \mathrm{~T}} \\
& \left\{\mathrm{e}^{\theta \mathrm{T}_{1}}-\theta \mathrm{T}_{1}-1\right\}-\frac{\mathrm{c} \alpha \beta(\beta+1) \mathrm{p}^{-\beta-2}}{\theta \mathrm{T}}\left\{\mathrm{e}^{\theta \mathrm{T}_{1}}-\theta \mathrm{T}_{1}-1\right\} \\
& -\frac{\mathrm{S} \alpha \beta(\beta+1) \mathrm{p}^{-\beta-2}}{\mathrm{~T}}\left\{\frac{\mathrm{T}^{2}+\mathrm{T}_{1}^{2}}{2}-\mathrm{TT}_{1}\right\}-\frac{2 \mathrm{U}_{1}^{2} \mathrm{Ic}_{1}}{\mathrm{p}^{3} \mathrm{~T}^{2}} \\
& \left\{\mathrm{e}^{\theta\left(\mathrm{T}_{1}-\mathrm{M}\right)}+\theta\left(\mathrm{M}-\mathrm{T}_{1}\right)-1\right\}-\frac{\alpha \beta(1-\beta) \mathrm{p}^{-\beta-1} \mathrm{IeM}^{2}}{2 \mathrm{~T}}
\end{aligned}
$$

$$
\begin{aligned}
& \mathrm{t}_{2.2}=\frac{\partial^{2}}{\partial \mathrm{T}_{1}^{2}} \mathrm{Z}_{2.2}\left(\mathrm{p}, \mathrm{T}_{1}\right)=-\frac{h \alpha \mathrm{p}^{-\beta} \mathrm{e}^{\theta \mathrm{T}_{\mathrm{T}}}}{\mathrm{T}}-\frac{\mathrm{c} \alpha \mathrm{p}^{-\beta} \theta \mathrm{e}^{\theta \mathrm{T}_{1}}}{\mathrm{~T}} \\
& -\frac{\mathrm{S} \alpha \mathrm{p}^{-\beta}}{\mathrm{T}}-\frac{\mathrm{U}_{1}^{2} \mathrm{I} \mathrm{c}_{1}}{\mathrm{pT}} \mathrm{e}^{\theta\left(\mathrm{T}_{1}-\mathrm{M}\right)} \\
& =\frac{\alpha \mathrm{p}^{-\beta}}{\mathrm{T}}(\mathrm{h}+\mathrm{c} \theta) \mathrm{e}^{\theta \mathrm{T}_{1}}-\frac{S \alpha \mathrm{p}^{-\beta}}{\mathrm{T}}-\frac{\mathrm{U}_{1}^{2} \mathrm{I} \mathrm{c}_{1}}{\mathrm{pT}} \mathrm{e}^{\theta\left(\mathrm{T}_{1}-\mathrm{M}\right)} \\
& \mathrm{s}_{2.2}=\frac{\partial^{2}}{\partial \mathrm{T}_{1} \partial \mathrm{p}} \mathrm{Z}_{2.2}\left(\mathrm{p}, \mathrm{T}_{1}\right)=\frac{\mathrm{h} \alpha \beta \mathrm{p}^{-\beta-1}}{\theta \mathrm{T}}\left\{\mathrm{e}^{\theta \mathrm{T}_{1}}-1\right\}+\frac{\mathrm{c} \alpha \beta \mathrm{p}^{-\beta-1}}{\mathrm{~T}} \\
& \left\{\mathrm{e}^{\theta \mathrm{T}_{1}}-1\right\}+\frac{\mathrm{S} \alpha \beta \mathrm{p}^{-\beta-1}}{\mathrm{~T}}\left(\mathrm{~T}_{1}-\mathrm{T}\right)+\frac{\mathrm{U}_{1}^{2} \mathrm{I} \mathrm{c}_{1}}{\mathrm{p}^{2} \mathrm{~T} \theta}\left\{\mathrm{e}^{\theta\left(\mathrm{T}_{1}-\mathrm{M}\right)}-1\right\}
\end{aligned}
$$

Case 3: $T_{1} \geq N$ : Based on the total purchased cost, CQ, total money $\mathrm{pD}(\mathrm{p}) \mathrm{M}+\mathrm{IE}_{2}$ in account at $\mathrm{M}$ and total money $\mathrm{pD}(\mathrm{p}) \mathrm{N}+\mathrm{IE}_{2}$ at $\mathrm{N}$, there are three sub cases may arise:

Sub Case 3.1: Let $\mathrm{pD}(\mathrm{p}) \mathrm{M}+\mathrm{IE}_{2} \geq \mathrm{CQ}$

This sub case is same as sub case 2.1; here sub case 3.1 designate decision variables and objective function.

Sub Case 3.2: Let $\mathrm{pD}(\mathrm{p}) \mathrm{M}+\mathrm{IE}_{2} \geq \mathrm{CQ}$ and

$$
\begin{aligned}
& \mathrm{pD}(\mathrm{p}) \cdot(\mathrm{N}-\mathrm{M})+\frac{\mathrm{pD}(\mathrm{p}) \cdot(\mathrm{N}-\mathrm{M})^{2} \mathrm{Ie}}{2} \\
& <\mathrm{CQ}-\left[\mathrm{pD}(\mathrm{p}) \cdot \mathrm{M}+\mathrm{IE}_{2}\right]
\end{aligned}
$$

This sub case is similar to sub case 2.2; here sub case 3.2 designate decision variables and objective function.

Sub Case 3.3: Let $\mathrm{pD}(\mathrm{p}) \cdot \mathrm{N}+\frac{\mathrm{pD}(\mathrm{p}) \cdot \mathrm{IeN}^{2}}{2}<\mathrm{CQ}$ : 
And:

$$
\begin{aligned}
& \mathrm{pD}(\mathrm{p}) \cdot(\mathrm{N}-\mathrm{M})+\frac{\mathrm{pIeD}(\mathrm{p}) \cdot(\mathrm{N}-\mathrm{M})^{2}}{2} \\
& <\mathrm{CQ}-\left[\mathrm{pD}(\mathrm{p}) \cdot \mathrm{M}+\mathrm{IE}_{2}\right]
\end{aligned}
$$

Here, retailer does not have enough money to pay off total purchase cost at N. He will do payment of $\mathrm{pD}$ $(\mathrm{p})+\mathrm{IE}_{2}$ at $\mathrm{M}$ and $\mathrm{pD}(\mathrm{p}) \cdot(\mathrm{N}-\mathrm{M})+\frac{\mathrm{pD}(\mathrm{p}) \mathrm{Ie} \cdot(\mathrm{N}-\mathrm{M})^{2}}{2}$ at $\mathrm{N}$. So, he has to pay interest on unpaid balance $\mathrm{U}_{1}=\mathrm{CQ}-\left[\mathrm{pD}(\mathrm{p}) \cdot \mathrm{M}+\mathrm{IE}_{2}\right]$ with $\mathrm{Ic}_{1}$ interest rate during (M, N) $\mathrm{U}_{2}=\mathrm{U}_{1}-\left[\mathrm{pD}(\mathrm{p}) .(\mathrm{N}-\mathrm{M})+\frac{\mathrm{pIeD}(\mathrm{p})(\mathrm{N}-\mathrm{M})^{2}}{2}\right]$ interest rate $\mathrm{Ic}_{2}$ during $\left(\mathrm{N}, \mathrm{T}_{1}\right)$.

Therefore, total interest charged on retailer; $\mathrm{IC}_{3 \square 3}$ per unit time is Eq. 36:

$$
\begin{aligned}
& \mathrm{Ic}_{3.3}=\frac{\mathrm{U}_{1} \mathrm{Ic}_{1}(\mathrm{~N}-\mathrm{M})}{\mathrm{T}}+\frac{\mathrm{U}_{2}^{2} \mathrm{Ic}_{2}}{\mathrm{PD}(\mathrm{p}) \mathrm{T}} \int_{\mathrm{N}}^{\mathrm{T}_{\mathrm{T}}} \mathrm{Q}(\mathrm{t}) \mathrm{dt} \\
& \mathrm{Ic}_{3.3}=\frac{\mathrm{U}_{1} \mathrm{Ic}_{1}(\mathrm{~N}-\mathrm{M})}{\mathrm{T}}+\frac{\mathrm{U}_{2}^{2} \mathrm{Ic}_{2}}{\mathrm{P} \theta \mathrm{T}}\left[\mathrm{e}^{\theta\left(\mathrm{T}_{1}-\mathrm{N}\right)}-\theta\left(\mathrm{T}_{1}-\mathrm{N}\right)-1\right]
\end{aligned}
$$

Interest earned per unit time is:

$$
\mathrm{IE}_{3.3}=\frac{\mathrm{IE}_{2}}{\mathrm{~T}}=\frac{\mathrm{I}_{\mathrm{e}} \mathrm{M}^{2} \alpha \mathrm{p}^{1-\beta}}{2 \mathrm{~T}}
$$

The net profit is:

$$
\begin{aligned}
& \mathrm{Z}_{3.3}\left(\mathrm{p} \cdot \mathrm{T}_{1}\right)=\mathrm{GR}-\mathrm{OC}-\mathrm{HC}-\mathrm{DC}-\mathrm{SC}-\mathrm{IC}_{3.3}+\mathrm{IE}_{3.3} \\
& =(\mathrm{p}-\mathrm{c}) \alpha \mathrm{p}^{-\beta}-\frac{\mathrm{A}}{\mathrm{T}}-\frac{\mathrm{hD}(\mathrm{p})}{\theta^{2} \mathrm{~T}}\left\{\mathrm{e}^{\theta \mathrm{T}_{1}}-\theta \mathrm{T}_{1}-1\right\}-\frac{\mathrm{cD}(\mathrm{p})}{\theta \mathrm{T}} \\
& \left\{\mathrm{e}^{\theta \mathrm{T}_{1}}-\theta \mathrm{T}_{1}-1\right\}-\frac{\mathrm{S} \alpha \mathrm{D}(\mathrm{p})}{\mathrm{T}}\left\{\frac{\mathrm{T}^{2}+\mathrm{T}_{1}^{2}}{2}-\mathrm{TT}_{1}\right\} \\
& -\frac{\mathrm{U}_{1} \mathrm{Ic}_{1}(\mathrm{~N}-\mathrm{M})}{\mathrm{T}}+\frac{\mathrm{U}_{2}^{2} \mathrm{Ic}_{2}}{\mathrm{p} \theta \mathrm{T}}\left[\begin{array}{l}
\mathrm{e}^{\theta\left(\mathrm{T}_{1}-\mathrm{M}\right)} \\
-\theta\left(\mathrm{T}_{1}-\mathrm{M}\right)-1
\end{array}\right]+\frac{\alpha \mathrm{p}^{1-\beta} \mathrm{IeM}^{2}}{2 \mathrm{~T}}
\end{aligned}
$$

The optimum values of $\mathrm{p}=\mathrm{p}_{3.3}$ and $\mathrm{T}_{1}=\mathrm{T}_{3.3}$ are solutions of Eq. 37:

$$
\begin{aligned}
& \frac{\partial}{\partial \mathrm{T}_{1}} \mathrm{Z}_{3.3}\left(\mathrm{p}, \mathrm{T}_{1}\right)=-\frac{\mathrm{hD}(\mathrm{p})}{\theta \mathrm{T}}\left\{\mathrm{e}^{\theta \mathrm{T}_{1}}-1\right\}-\frac{c \alpha \mathrm{p}^{-\beta}}{\mathrm{T}}\left\{\mathrm{e}^{\theta \mathrm{T}_{1}}-1\right\} \\
& -\frac{\alpha \mathrm{SD}(\mathrm{p})}{\mathrm{T}}\left\{\mathrm{T}_{1}-\mathrm{T}\right\}+\frac{\mathrm{U}_{2}^{2} \mathrm{Ic}_{2}}{\mathrm{pT}}\left\{\mathrm{e}^{\theta\left(\mathrm{T}_{1}-\mathrm{N}\right)}-1\right\}=0
\end{aligned}
$$

And:

$$
\begin{aligned}
& \frac{\partial}{\partial \mathrm{p}} \mathrm{Z}_{3.3}\left(\mathrm{p}, \mathrm{T}_{1}\right)=\alpha \mathrm{p}^{-\beta}-\alpha \beta(\mathrm{p}-\mathrm{c}) \mathrm{p}^{-\beta-1}+\frac{h \alpha \beta \mathrm{p}^{-\beta-1}}{\theta^{2} \mathrm{~T}} \\
& \left\{\mathrm{e}^{\theta \mathrm{T}_{1}}-\theta \mathrm{T}_{1}-1\right\}+\frac{\mathrm{c} \alpha \beta \mathrm{p}^{-\beta-1}}{\theta \mathrm{T}}\left\{\mathrm{e}^{\theta \mathrm{T}_{1}}-\theta \mathrm{T}_{1}-1\right\} \\
& +\frac{\mathrm{S} \alpha \beta \mathrm{p}^{-\beta-1}}{\mathrm{~T}}\left\{\frac{\mathrm{T}^{2}+\mathrm{T}_{1}^{2}}{2}-\mathrm{TT}_{1}\right\}-\frac{\mathrm{U}_{2}^{2} \mathrm{Ic}_{2}}{\mathrm{p}^{2} \mathrm{~T} \theta} \\
& \left\{\mathrm{e}^{\theta\left(\mathrm{T}_{1}-\mathrm{N}\right)}-\theta\left(\mathrm{T}_{1}-\mathrm{N}\right)-1\right\}+\frac{\alpha(1-\beta) \mathrm{p}^{-\beta} \mathrm{IeM}^{2}}{2 \mathrm{~T}}=0
\end{aligned}
$$

To maximize the net profit, provided:

$$
\mathrm{r}_{3.3} \mathrm{t}_{3.3}-\mathrm{s}_{3.3}^{2}<0
$$

$$
\begin{aligned}
& \mathrm{r}_{3.3}=\frac{\partial^{2}}{\partial \mathrm{p}^{2}} \mathrm{Z}_{3.3}\left(\mathrm{p}, \mathrm{T}_{1}\right)=-\alpha \beta(\beta-1) \mathrm{p}^{-\beta-1}-\alpha \beta(\beta+1) \mathrm{cp}^{-\beta-2} \\
& -\frac{\mathrm{h} \alpha \beta(\beta+1)}{\theta^{2} \mathrm{~T}}\left\{\mathrm{e}^{\theta \mathrm{T}_{1}}-\theta \mathrm{T}_{1}-1\right\}-\frac{\mathrm{c} \alpha \beta(\beta+1)}{\theta \mathrm{T}}\left\{\mathrm{e}^{\theta \mathrm{T}_{1}}-\theta \mathrm{T}_{1}-1\right\} \\
& -\frac{\mathrm{S} \alpha \beta(\beta+1)}{\mathrm{T}}\left\{\frac{\mathrm{T}^{2}+\mathrm{T}_{1}^{2}}{2}-\mathrm{TT}_{1}\right\}+\frac{2 \mathrm{U}_{2}^{2} \mathrm{Ic}_{2}}{\mathrm{p}^{3} \mathrm{~T} \theta} \\
& \left\{\mathrm{e}^{\theta\left(\mathrm{T}_{1}-\mathrm{N}\right)}-\theta\left(\mathrm{T}_{1}-\mathrm{N}\right)-1\right\}+\frac{\alpha \beta(\beta-1) \mathrm{p}^{-\beta-1} \mathrm{IeM}^{2}}{2 \mathrm{~T}} \\
& \mathrm{t}_{3.3}=\frac{\partial^{2}}{\partial \mathrm{T}_{1}^{2}} \mathrm{Z}_{3.3}\left(\mathrm{p}, \mathrm{T}_{1}\right)=-\frac{(\mathrm{h}+\mathrm{c} \theta) \alpha \mathrm{p}^{-\beta} \mathrm{e}^{\theta \mathrm{T}_{1}}}{\mathrm{~T}} \\
& -\frac{\mathrm{S} \alpha^{2} \mathrm{p}^{-\beta}}{\mathrm{T}}+\frac{\mathrm{U}_{2}^{2} \mathrm{Ic}_{2}}{\mathrm{pT}} \theta \mathrm{e}^{\theta\left(\mathrm{T}_{1}-\mathrm{N}\right)}
\end{aligned}
$$

And:

$$
\begin{aligned}
& \mathrm{s}_{3.3}=\frac{\partial^{2}}{\partial \mathrm{T}_{1} \partial \mathrm{p}} \mathrm{Z}_{3.3}\left(\mathrm{p}, \mathrm{T}_{1}\right)=\frac{\mathrm{h} \alpha \beta \mathrm{p}^{-\beta-1}}{\theta \mathrm{T}}\left\{\mathrm{e}^{\theta \mathrm{T}_{1}}-1\right\} \\
& +\frac{\mathrm{c} \alpha \beta \mathrm{p}^{-\beta-1}}{\mathrm{~T}}\left\{\mathrm{e}^{\theta \mathrm{T}_{1}}-1\right\}+\frac{\mathrm{S} \alpha \beta \mathrm{p}^{-\beta-1}}{\mathrm{~T}}\left(\mathrm{~T}_{1}-\mathrm{T}\right) \\
& -\frac{\mathrm{U}_{2}^{2} \mathrm{Ic}_{2}}{\mathrm{p}^{2} \mathrm{~T}}\left\{\mathrm{e}^{\theta\left(\mathrm{T}_{1}-\mathrm{N}\right)}-1\right\}
\end{aligned}
$$

Algorithm for optimal solution: Step 1: Compute $\mathrm{T}_{1}=$ $\mathrm{T}_{1}{ }^{*}$ and $\mathrm{p}=\mathrm{o}_{1}$ from case- 1 :

Step 2: If $\mathrm{T}_{1}<\mathrm{M}$.

Then calculate:

$$
\mathrm{Z}\left(\mathrm{p}, \mathrm{T}_{1}\right)=\max \left\{\mathrm{Z}_{\mathrm{i}}\left(\mathrm{p}, \mathrm{T}_{1}\right)\right\} \text { Where } \mathrm{I}=1,2.1,2.2,3.1,
$$

3.2, 3.3.

Step 3: If $\mathrm{M}<\mathrm{T}_{1}<\mathrm{N}$. 
If $\mathrm{pD}(\mathrm{p}) \mathrm{M}+\mathrm{IE}_{2} \geq \mathrm{CQ}$ is true then compute $\mathrm{T}_{1}=\mathrm{T}_{2.1}$ and $\mathrm{p}=\mathrm{p}_{2.1}$ from sub case 2.1 or $\mathrm{T}_{1}=\mathrm{T}_{3.1}$ and $\mathrm{p}=\mathrm{p}_{3.1}$ from sub case 3.1, repeat step 2 and stop.

If $\mathrm{pD}(\mathrm{p}) \mathrm{M}+\mathrm{IE}_{2} \geq \mathrm{CQ}$ is not true but $\mathrm{pD}(\mathrm{p})$

$$
M+\mathrm{IE}_{2}<\mathrm{CQ} \text { and } \begin{aligned}
& \mathrm{pD}(\mathrm{p})(\mathrm{N}-\mathrm{M})+\frac{\mathrm{pIeD}(\mathrm{p})(\mathrm{N}-\mathrm{M})^{2}}{2} \geq \\
& \mathrm{cD}(\mathrm{p}) \mathrm{T}-\mathrm{pD}(\mathrm{p}) \mathrm{T}-\frac{\mathrm{pIeD}(\mathrm{p}) \mathrm{M}^{2}}{2}
\end{aligned} \text {, then }
$$

compute $\mathrm{T}_{1}=\mathrm{T}_{2.1}$ and $\mathrm{p}=\mathrm{p}_{2.2}$ from subcase 2.2 or $\mathrm{T}_{1}=$ $\mathrm{T}_{3.2}$ and $\mathrm{p}=\mathrm{p}_{3.2}$ from subcase 3.2.

If $\mathrm{pD}$ (p) $\mathrm{M}+\mathrm{IE}_{2} \geq \mathrm{CQ}$ is not true, but $\mathrm{pD}$ (p)

$$
M+\mathrm{IE}_{2}<\mathrm{CQ} \text { and } \begin{aligned}
& \mathrm{pD}(\mathrm{p})(\mathrm{N}-\mathrm{M})+\frac{\mathrm{pIeD}(\mathrm{p})(\mathrm{N}-\mathrm{M})^{2}}{2} \geq \\
& \mathrm{cD}(\mathrm{p}) \mathrm{T}-\mathrm{pD}(\mathrm{p}) \mathrm{T}-\frac{\mathrm{pIeD}(\mathrm{p}) \mathrm{M}^{2}}{2}
\end{aligned} \text { is not }
$$

true, then compute $\mathrm{T}_{1}=\mathrm{T}_{3.3}$ and $\mathrm{p}=\mathrm{p}_{3.3}$ from sub case 3.3 , repeat step 2 and stop.

Step 4: $\mathrm{M}<\mathrm{T}_{1}<\mathrm{N}$ is not true then computes $\mathrm{T}_{1}=\mathrm{T}_{3.3}$ and $\mathrm{p}=\mathrm{p}_{3.3}$ from sub case 3.3 , repeat step 2 and stop.

Numerical examples: The preceding theory can be illustrated by the following numerical example where the parameters are given as follows:

Demand parameter, $\alpha=10,000 \quad \beta=2.5$, Selling price, $\mathrm{p}=13$, Deterioration rate, $\theta=0.03$,

Deterioration cost $\mathrm{C}=0.05$, Shortage cost $\mathrm{S}=3$, Holding cost $h=2.5$, First delay period, $\mathrm{M}=0.08$, Sec delay period, $\mathrm{N}=0.1$, the interest earned, $\mathrm{Ie}=0.05$, the interest charged, Ic1 $=0.12$, the interest charged, Ic2 = 0.20 (Ic1 > Ic2), $\mathrm{T}=10$.

Table 1: Retailer does not pay any interest to the supplier

\begin{tabular}{llll}
\hline $\mathrm{n}$ & $\mathrm{T}_{1}$ & $\mathrm{P}$ & Profit $\mathrm{Z}\left(\mathrm{p}, \mathrm{T}_{1}\right)$ \\
\hline 1 & 0.822346 & 0.126863 & 43.8315 \\
2 & 0.856542 & 0.128116 & 52.8059 \\
3 & 0.883456 & 0.128522 & 57.2229 \\
4 & 0.917391 & 0.128723 & 63.8578 \\
5 & 0.952656 & 0.128844 & 76.8586 \\
\hline
\end{tabular}

Table 2: Retailer will have to pay interest on unpaid balance at the rate of interest $\mathrm{Ic}_{1}$; Retailer does not have enough money to pay off at $\mathrm{M}$

\begin{tabular}{llll}
\hline $\mathrm{n}$ & $\mathrm{T}_{1}$ & $\mathrm{p}$ & Profit $\mathrm{Z}\left(\mathrm{p}, \mathrm{T}_{1}\right)$ \\
\hline 1 & 0.74453 & 0.137546 & 35.2673 \\
2 & 0.78238 & 0.139723 & 39.2645 \\
3 & 0.82874 & 0.143271 & 46.2451 \\
4 & 0.85961 & 0.151293 & 48.2103 \\
5 & 0.89453 & 0.157934 & 52.3940 \\
\hline
\end{tabular}

Table 3: Retailer pays interest at the rate of $\mathrm{Ic}_{2}$ to the supplier; Retailer does not have enough money to pay off at $\mathrm{N}$

\begin{tabular}{llll}
\hline $\mathrm{n}$ & $\mathrm{T}_{1}$ & $\mathrm{p}$ & Profit $\mathrm{Z}\left(\mathrm{p}, \mathrm{T}_{1}\right)$ \\
\hline 1 & 0.79343 & 0.132163 & 30.7830 \\
2 & 0.82871 & 0.138945 & 36.6734 \\
3 & 0.83456 & 0.145280 & 40.0989 \\
4 & 0.86820 & 0.150834 & 43.7824 \\
5 & 0.87832 & 0.153278 & 54.9702 \\
\hline
\end{tabular}

Using the above algorithm, we obtain the computational results as shown in Table 1-3.

Results: The data obtained clearly shows that individual optimal solutions are very different from each other. However, there exists a solution which ultimately provides the Maximize the total profit operating of inventory system. In the above tables, it is observed that as the value of $\mathrm{T}_{1}$ and $\mathrm{p}$ are increased and then the total cost is increased. Thus, the optimal solution of the problem is $\mathrm{Z}\left(\mathrm{p}, \mathrm{T}_{1}\right)=76.8586 \mathrm{at}\left(\mathrm{p}, \mathrm{T}_{1}\right)$ $=(0.952656,0.128844)$.

\section{CONCLUSION}

In this study, we introduced a new idea of trade credits, namely, the supplier charges the retailer progressive interest rates if the retailer prolongs its unpaid balance. By offering progressive interest rates to the retailers, a supplier, can secure competitive market advantage over the competitors and possibly improve market share profit.

Shortages are allowed and completely backlogged in the present model. In many practical situations, stock out is unavoidable due to various uncertainties. There are many situations in which the profit of the stored item is higher than its back order cost. Consideration of shortages is economically desirable in these cases. The traditional parameters of holding cost is assumed here to be time varying. As the changes in the time value of money and in the price, index, holding cost cannot remain constant over time. It is assumed that the holding cost is linearly increasing function of time.

We developed theoretical results to obtain the optimal replenishment interval by examine the explicit condition. We proposed an algorithm to find the optimal ordering policy. A numerical study has been performed to observe the sensitivity of the effect of demand parameter changes. Further, the model can be enriched by incorporating other realistic parameters such as Weibull distribution deterioration rate, inflation rate, partial backlogging and in progressive interest charges.

\section{REFERENCES}

Aggarwal, S.P. and C.K. Jaggi, 1995. Ordering policies of deteriorating items under permissible delay in payments. J. Oper. Res. Soc., 46: 658-662.

Chang, C.T., J.T. Teng and S.K. Goyal, 2010. Optimal replenishment policies for non-instantaneous deteriorating items with stock-dependent demand. Int. J. Product. Econ., 123: 62-68. DOI: 10.1016/j.ijpe.2009.06.042 
Chang, H.J. and C.Y. Dye, 2001. An inventory model for deteriorating items with partial backlogging and permissible delay in payments. Int. J. Syst. Sci., 32: 345-352. DOI: 10.1080/002077201300029700

Chung, K.J. and S.F. Tsai, 2001. Inventory systems for deteriorating items with shortages and a linear trend in demand-taking account of time value. Comput. Oper. Res., 28: 915-934. DOI: 10.1016/S0305-0548(00)00016-2

Goyal, S.K., 1985. Economic order quantity under conditions of permissible delay in payments. J Oper. Res. Soc., 36: 35-38.

Goyal, S.K., J.T. Teng and C.T. Chang, 2007. Optimal ordering policies when the supplier provides a progressive interest scheme. Eur. J. Oper. Res., 179: 404-413. DOI: 10.1016/j.ejor.2006.03.037

Haley, C.W. and R.C. Higgins, 1973. Inventory policy and trade credit financing. Manage. Sci., 20: 464471.

Hwang, H. and S.W. Shinn, 1997. Retailer's pricing and lot sizing policy for exponentially deteriorating products under the condition of permissible delay in payments. Comput. Operat. Res., 24: 539-547. DOI: $10.1016 / \mathrm{S} 0305-0548(96) 00069-\mathrm{X}$

Jamal, A.M.M., B.R. Sarkar and S. Wang, 1997. An ordering policy for deteriorating items with allowable shortage and permissible delay in payment. J. Oper. Res. Soc., 48: 826-833.
Liao, H.C., C.H. Tsai and C.T. Su, 2000. An inventory model with deteriorating items under inflation when a delay in payment is permissible. Int. J. Product. Econ., 63: 207-214. DOI: 10.1016/S09255273(99)00015-8

Mandal, B.N. and S. Phaujdar, 1988. An inventory model for deteriorating items and stock dependent selling rate. Eur. J. Oper. Res. Soc., 40: 483-488.

Singh S.R., R.K. Pandey and K. Mukesh, 2008. Policy decisions for a stock-dependent demand rate inventory model with progressive permissible delay in payment. Int. Trans. Math. Sci. Comput., 1: 59-74.

Soni, H. and N.H. Shah, 2008. Optimal ordering policy for stock-dependent demand under progressive payment scheme. Eur. J. Operat. Res., 184: 91-100. DOI: 10.1016/j.ejor.2006.10.048

Teng, J.T., 2002. On the economic order quantity under conditions of permissible delay in payments. J. Oper. Res. Soc., 53: 915-918.

Teng, J.T., C.T. Chang and S.K. Goyal, 2005. Optimal pricing and ordering policy under permissible delay in payments. Int. J. Product. Econ., 97: 121129. DOI: $10.1016 /$ j.jpe.2004.04.010

Wee, H.M., 1995. A deterministic lot-size inventory model for deteriorating items with shortages and a declining market. Comput. Operat., 22: 345-356. DOI: 10.1016/0305-0548(94)E0005-R 